\title{
ERGODICITY OF STOCHASTICALLY FORCED LARGE SCALE GEOPHYSICAL FLOWS
}

\author{
JINQIAO DUAN and BENIAMIN GOLDYS
}

(Received 30 March 2001)

\begin{abstract}
We investigate the ergodicity of 2D large scale quasigeostrophic flows under random wind forcing. We show that the quasigeostrophic flows are ergodic under suitable conditions on the random forcing and on the fluid domain, and under no restrictions on viscosity, Ekman constant or Coriolis parameter. When these conditions are satisfied, then for any observable of the quasigeostrophic flows, its time average approximates the statistical ensemble average, as long as the time interval is sufficiently long.
\end{abstract}

2000 Mathematics Subject Classification. 37A25, 60H15, 76D05, 86A05.

1. Introduction. The models for geophysical flows are usually very complicated. Simplified models have been developed to investigate the basic key features of large scale phenomena. These models filter out undesired high frequency oscillations in geophysical flows and are derived at asymptotically high rotation rate or small Rossby number.

An important example of such a geophysical flow model is the quasigeostrophic flow model [14]

$$
\Delta \psi_{t}+J(\psi, \Delta \psi)+\beta \psi_{x}=v \Delta^{2} \psi-r \Delta \psi+\text { wind forcing }
$$

where $\psi(x, y, t)$ is the stream function, $\beta \geq 0$ is the meridional gradient of the Coriolis parameter, $v>0$ is the viscous dissipation constant, and $r>0$ is the Ekman dissipation constant. Moreover, $J(f, g)=f_{x} g_{y}-f_{y} g_{x}$ denotes the Jacobian operator.

The quasigeostrophic equation has been derived as an approximation of the rotating shallow water equations by the conventional asymptotic expansion in small Rossby number [14]. Recently, the randomly forced quasigeostrophic flow model has been used to study various phenomena in geophysical flows under uncertain wind forcing $[5,10,11,12,16]$.

Introducing (relative) vorticity $\omega(x, y, t)=\Delta \psi(x, y, t)$, the quasigeostrophic equation can be written as

$$
\omega_{t}+J(\psi, \omega)+\beta \psi_{x}=v \Delta \omega-r \omega+\text { wind forcing, }
$$

where $(x, y) \in D$ and $D \subset R^{2}$ denotes a bounded domain with sufficiently regular boundary. Potential vorticity is defined as $\omega+\beta y$. The boundary conditions are no normal flow $(\psi=0)$ and free-slip $(\omega=0)$ on $\partial D$ as in Pedlosky (see [15, page 34]) or 
in Dymnikov and Kazantsev [7]:

$$
\psi=\omega=0 \quad \text { on } \partial D
$$

An appropriate initial condition $\omega(0)$ is also imposed. We note that the Poincaré inequality holds with these boundary conditions.

An invariant measure for stochastic systems is like a "statistical steady state" and is a part of the asymptotic permanent regime of the system [1]. When there is only one invariant measure for the quasigeostrophic flows modeled by (2.3), we have the so-called ergodic principle, that is, for any observable of the quasigeostrophic flows, its time average on $[0, T]$ approaches the statistical ensemble average, as $T$ goes to infinity.

We will investigate the existence and uniqueness of invariant measures for quasigeostrophic flows. After reviewing the mathematical setup in Section 2, we study existence and uniqueness of invariant measures in Sections 3 and 4, respectively. Finally, we summarize our results in Section 5.

2. Mathematical setup. In the following we use the abbreviations $H=L^{2}(D), H_{0}^{k}=$ $H_{0}^{k}(D), H^{k}=H^{k}(D), 0<k<\infty$, for the standard Sobolev spaces. Let $\langle\cdot, \cdot\rangle$ and $\|\cdot\|$ denote the standard scalar product and norm in $L^{2}$, respectively. Moreover, the norms for $H_{0}^{k}$ are denoted by $\|\cdot\|_{H^{k}}$. Due to the Poincaré inequality [8], $\|\Delta \varphi\|$ is an equivalent norm for $H_{0}^{2}$. It is well known that the linear operator

$$
A=v \Delta: H \longrightarrow H
$$

with domain $D(A)=H^{2} \cap H_{0}^{1}$ is selfadjoint. Note that $A$ generates a strongly continuous, and in fact, an analytic semigroup $S(t)$ on $L^{2}$ (see [13]). The spectrum of $A$ consists of eigenvalues $0>\lambda_{1}>\lambda_{2} \geq \lambda_{3} \geq \cdots$ with corresponding normalized eigenfunctions $e_{1}, e_{2}, \ldots$. The set of these eigenfunctions is complete in $L^{2}$. For example, for the square domain $D=(0,1) \times(0,1)$ the eigenvalues are given by $-v\left(m^{2}+n^{2}\right) \pi^{2}$ for positive integers $m, n$, and the associated eigenfunctions are suitable multiples of $\sin (m \pi x) \sin (n \pi y)$.

We define the nonlinear operator $F$ by

$$
F(\omega)=-r \omega-\beta \psi_{x}-J(\psi, \omega)
$$

then (1.2) can be rewritten as the abstract evolution equation together with the initial condition

$$
\begin{gathered}
d \omega=(A \omega+F(\omega)) d t+\sqrt{Q} d W, \\
\omega(0) \text { is given, }
\end{gathered}
$$

where $W(x, y, t)$ is a Wiener process defined on a probability space $(\Omega, \mathscr{F}, \mathbb{P})$. The covariance operator $Q: H \rightarrow H$ for this Wiener process is a nonnegative and symmetric linear continuous operator to be specified below. The term with Ito derivative, $\sqrt{Q} d W$, is a model for the white-in-time noise representing the random wind forcing. This 
equation can be rewritten in the mild (integral) form

$$
\omega(t)=S(t) \omega(0)+\int_{0}^{t} S(t-s) F(\omega(s)) d s+Z(t),
$$

where $Z(t)$ is the stochastic convolution

$$
Z(t)=\int_{0}^{t} S(t-s) \sqrt{Q} d W(s), \quad t>0 .
$$

In fact, $Z(t)$ is an Ornstein-Uhlenbeck process and it is the solution of the linearized version of (2.3):

$$
d Z=A Z d t+\sqrt{Q} d W .
$$

In this paper, we always assume that the covariance operator $Q$ for the Wiener process $W(t)$ is of trace class, that is, Trace $Q<+\infty$. Thus we only consider the noise that is white in time but colored in space. Then the stochastic convolution $Z(t)$ has a continuous version with values in $H=L^{2}(D)$ (see [3, Theorem 5.14]).

We can specifically define an appropriate class of Wiener processes $W(t)$ satisfying the above condition. Let $\beta_{k}(t)$, for positive integer $k$, denote a family of independent real-valued Brownian motions. Furthermore, choose positive constant $\alpha_{k}$ such that

$$
\sum_{k=1}^{\infty} \frac{\alpha_{k}^{2}}{\left|\lambda_{k}\right|^{1-\gamma}}<\infty,
$$

for some $0<\gamma<1$. Then we define the white noise by

$$
\sqrt{Q} \dot{W}(t):=\sum_{k=1}^{\infty} \alpha_{k} \dot{\beta}_{k}(t) e_{k}, \quad t \geq 0 .
$$

Note that the eigenvalues $\lambda_{k}$ for the operator $A$ behave like $k$ in two dimensions and also note that the Riemann zeta function $\zeta(s)=\sum_{k=1}^{\infty} 1 / k^{s}$ is well defined for $s>1$. We see that condition (2.8) is satisfied when $k^{-1 / 2} \leq \alpha_{k} \leq k^{-3 / 8}$.

We further assume that

$$
\kappa(D)=\inf _{0<\rho<\operatorname{diam}(D)} \inf _{(x, y) \in D} \frac{\operatorname{meas}(D \cap B(x, y ; \rho))}{\rho^{2}}>0,
$$

where diam $(D)$ is the diameter of $D$ (the least upper bound of two-point distances in $D)$, meas $(\cdot)$ denotes the Lebesgue measure, and $B(x, y ; \rho)$ is the open disk centered at $(x, y)$ and with radius $\rho$. We also assume that the eigenfunctions $e_{k}$ satisfy

$$
e_{k} \in C_{0}(\bar{D}), \quad\left|e_{k}(x, y)\right| \leq C, \quad\left|\partial_{x} e_{k}(x, y)\right|, \quad\left|\partial_{y} e_{k}(x, y)\right| \leq C \sqrt{\left|\lambda_{k}\right|},
$$

for $(x, y) \in D$, positive integer $k$, and some constant $C>0$. For the square domain $D=(0,1) \times(0,1)$, these conditions are all satisfied. Then, according to [4, Theorem 5.2.9], the stochastic convolution $Z(t)$ has a continuous version with values in $L^{2}(D)$. (Actually, in this case, $Z(t)$ is in $C_{0}(D)$, the Banach space of continuous functions satisfying the zero Dirichlet boundary condition on $D$.) For this Wiener process $W(t)$ 
in (2.9), the stochastic convolution $Z(t)$ is

$$
Z(t)=\sum_{k=1}^{\infty} \alpha_{k} e_{k} \int_{0}^{t} e^{-\lambda_{k}(t-s)} d \beta_{k}(s), \quad t \geq 0 .
$$

As shown in [2], for every initial condition $\omega(0) \in L^{2}(D)$, there exists a unique global mild solution $\omega(x, y, t)$ of the quasigeostrophic flow model (2.3). This solution is in $C\left([0, T] ; L^{2}(D)\right)$ for every $T>0$.

3. Existence of an invariant measure. Now we consider invariant measure for the quasigeostrophic flow model (2.3). For the rest of the paper, we denote $\omega(t ; x)$ as the solution of the quasigeostrophic flow model with initial condition (not the spatial point) $x \in H$.

We introduce the usual notations. The Markovian transition semigroup is

$$
\left(P_{t} g\right)(x)=\mathbb{E}[g(\omega(t ; x))],
$$

for $g \in B_{b}(H)$, the space of bounded Borel measurable functions. Hereafter $\mathbb{E}$ is the expectation. The transition probability is

$$
P_{t}(x, \Gamma)=\mathbb{P}(\omega(t ; x) \in \Gamma),
$$

for $x \in H$ and $\Gamma \in \mathscr{B}(H)$, the $\sigma$-algebra of Borel sets in $H$.

A probability measure $\mu$ on $(H, \mathscr{B}(H))$ is called invariant if

$$
\int g d \mu=\int P_{t} g d \mu
$$

for any $t>0$ and $g \in B_{b}(H)$, or, equivalently,

$$
\int_{H} P_{t}(x, \Gamma) d \mu=\mu(\Gamma)
$$

for any $t>0, x \in H$ and $\Gamma \in \mathscr{B}(H)$.

The existence of an invariant measure for the quasigeostrophic flow model (2.3) follows from a tightness or, equivalently, a compactness argument [17]. If the meansquare norm of the solution is bounded for all time $t>0$ and for all initial data, then by the Chebyshev inequality, the solution is bounded in probability, which further implies that the family of measures on $(H, \mathscr{B}(H))$

$$
\frac{1}{T} \int_{0}^{T} P_{t}(x, \cdot) d t, \quad T \geq 1,
$$

is tight for some $x \in H$ (see [4, pages 89-90]). Thus by [4, Corollary 3.1.2], there exists an invariant measure for the quasigeostrophic flow model (2.3). So in the rest of this section, we estimate the mean-square norm $\mathbb{E}\|\omega(t)\|^{2}$. 
We assume that

$$
\int_{0}^{\infty}\|S(r) \sqrt{Q}\|_{H S}^{2} d r<+\infty
$$

where $\|\cdot\|_{H S}$ is the Hilbert-Schmidt norm. We rewrite (2.5) as

$$
\omega(t)=Y(t)+Z(t)
$$

where

$$
Y(t)=S(t) x+\int_{0}^{t} S(t-s) F(\omega(s)) d s
$$

with initial data $\omega(0)=x$, and $Z(t)$ is the Ornstein-Uhlenbeck process in (2.6).

By [3, Corollary 4.14], for any $x \in H$,

$$
\sup _{t \geq 0} \mathbb{E}\|Z(t)\|^{2}=\sup _{t \geq 0} \mathbb{E} \int_{0}^{t}\|S(r) \sqrt{Q}\|_{H S}^{2} d r<+\infty .
$$

By [2] or follow a Yosida approximation combined with $L^{2}$-norm estimate as in [4, Proposition 6.1.6], we have, for any $x \in H$,

$$
\sup _{t \geq 0} \mathbb{E}\|Y(t)\|^{2}<+\infty
$$

Note that

$$
\begin{aligned}
\|\omega(t)\|^{2} & =\langle Y+Z, Y+Z\rangle=\|Y\|^{2}+2\langle Y, Z\rangle+\|Z\|^{2} \\
& \leq\|Y\|^{2}+2\|Y\|\|Z\|+\|Z\|^{2} \\
& \leq 2\left(\|Y\|^{2}+\|Z\|^{2}\right) .
\end{aligned}
$$

Thus, by (3.9) and (3.10),

$$
\sup _{t \geq 0} \mathbb{E}\|\omega(t)\|^{2}<+\infty
$$

By the argument in the beginning of this section, there exists at least one invariant measure for the quasigeostrophic flow model (2.3). We have the main result in this section.

THEOREM 3.1. Assume that $\int_{0}^{+\infty}\|S(r) \sqrt{Q}\|_{H S}^{2} d r<+\infty$. Then there exists at least one invariant probability measure for the quasigeostrophic flow model (2.3) in the space $L^{2}(D)$ of square-integrable vorticities.

4. Uniqueness of an invariant measure. Now we consider the uniqueness of invariant measure for the quasigeostrophic flow model (2.3). As we know in [4, Chapter 4], the uniqueness of invariant measure is a consequence of regularity of the transition semigroup $P_{t}$, by the Doob's theorem. Due to Khasminskii's theorem, strong Feller and irreducibility properties imply the regularity. So we now try to prove the strong Feller and irreducibility properties for the transition semigroup $P_{t}$. 
Strong Feller property means that for every $g(x)$ in $B_{b}(H)$, the space of bounded Borel measurable functions on $H, P_{t} g(x)$ is in $C_{b}(H)$, the space of bounded continuous functions on $H$.

Irreducibility property means that for every Borel set in $H$, that is, for every $\Gamma$ in $\mathscr{B}(H), P_{t}(x, \Gamma)$ is positive for any $x \in H$ and $t>0$.

STRONG FELLER PROPERTY. We first consider strong Feller property. Note that (see [3, page 119])

$$
\text { Trace } \int_{0}^{t} S(r) Q S^{*}(r) d r=\int_{0}^{t}\|S(r) \sqrt{Q}\|_{H S}^{2} d r \text {. }
$$

So the condition for the existence of invariant measures in Theorem 3.1, that is, $\int_{0}^{+\infty}\|S(r) \sqrt{Q}\|_{H S}^{2} d r<+\infty$, implies that the linear integral operator $Q_{t}: H \rightarrow H$,

$$
Q_{t} x:=\int_{0}^{t} S(r) Q S^{*}(r) x d r, \quad x \in H
$$

is of trace class for any $t>0$.

We further assume that

$$
\text { Image } S(t) \subset \text { Image } Q_{t}^{1 / 2} \text {. }
$$

Then follow a similar argument as in the proofs of Theorem 7.2.4 in [4] and of Theorem 3.1 in [9], we conclude that $P_{t}, t>0$, is a strong Feller semigroup.

IRREDUCIBILITY PROPERTY. Now we consider irreducibility property. We further assume that the covariance operator $Q$ is one-to-one (or injective), that is, the kernel $\operatorname{ker} Q=\{0\}$. Then, as in the proof of Theorem 7.4.2 in [4] and of Theorem 3.1 in [9], $P_{t}, t>0$, is irreducible.

Thus, with the strong Feller and irreducibility properties proved above, using Doob's theorem [4, Theorem 4.2.1], there exists a unique invariant measure $\mu$ on $(H, \mathscr{B}(H))$, and all other transition probability measures $P_{t}(x, \cdot), x \in H$, approach this unique invariant measure $\mu$ as time goes to infinity.

Therefore, we have the following main theorem in this section.

THEOREM 4.1. Assume that

(i) $\int_{0}^{+\infty}\|S(r) \sqrt{Q}\|_{H S}^{2} d r<+\infty$,

(ii) Image $S(t) \subset$ Image $Q_{t}^{1 / 2}$, where $Q_{t}$ is defined in (4.2), and

(iii) the covariance operator $Q: L^{2}(D) \rightarrow L^{2}(D)$ is one-to-one.

Then

(A) there exists a unique invariant probability measure $\mu$ for the quasigeostrophic flow system (2.3) in the space $L^{2}(D)$ of square-integrable vorticities;

(B) moreover, for any $\omega \in L^{2}(D)$, the transition probability measures $P_{t}(\omega, \cdot)$ approach the unique invariant probability measure $\mu$. Namely, for any $\Gamma \in \mathscr{B}(H)$,

$$
\lim _{t \rightarrow+\infty} P_{t}(\omega, \Gamma)=\mu(\Gamma)
$$


(C) quasigeostrophic flow system (2.3) is ergodic, namely,

$$
\lim _{T \rightarrow+\infty} \int_{0}^{T} g(\omega(t)) d t=\int_{L^{2}} g d \mu, \quad \mathbb{P}-\text { a.s. }
$$

for all solution $\omega(t)$ with initial date in $L^{2}(D)$ and all Borel measurable function $g: L^{2}(D) \rightarrow \mathbb{R}$ such that $\int_{L^{2}(D)}\|g\| d \mu<\infty$.

The ergodicity in part $(\mathrm{C})$ above is a consequence of the uniqueness of the invariant measure $\mu$ (see [4, Theorem 3.2.6]).

5. Summary. In this paper, we have studied ergodicity of large scale quasigeostrophic flows under random wind forcing. We have shown that the quasigeostrophic flows are ergodic under suitable conditions on the random forcing and on the fluid domain, and under no restrictions on viscosity, Ekman constant or Coriolis parameter. When these conditions are satisfied, then for any observable of the quasigeostrophic flows, its time average approximates the statistical ensemble average, as long as the time interval is sufficiently long.

There is recent work on random dynamical attractors for the quasigeostrophic flow model by Duan et al. [6]. A consequence of that work implies that, when viscosity is sufficiently large and when the trace of the covariance operator for the Wiener process is sufficiently small, then all quasigeostrophic motions approach a point random attractor exponentially fast as time goes to infinity. This is a very rare case. This point random attractor corresponds to a unique invariant Dirac measure, that is, the supporting point of the Dirac measure is a global (point) attractor, and thus under these conditions, quasigeostrophic flows are also ergodic. These conditions are different from the ergodic conditions in the current paper. For example, in the current paper, we do not impose any condition on viscosity, or on the size of the trace of the covariance operator for the Wiener process.

ACKNOWLEDGEMENTS. A part of this work was done while J. Duan was visiting the University of New South Wales, Australia. This work was partly supported by the Australia Research Council and by the NSF Grant DMS-9973204.

\section{REFERENCES}

[1] L. Arnold, Random Dynamical Systems, Springer Monographs in Mathematics, SpringerVerlag, Berlin, 1998. MR 2000m:37087. Zbl 0906.34001.

[2] J. R. Brannan, J. Duan, and T. Wanner, Dissipative quasi-geostrophic dynamics under random forcing, J. Math. Anal. Appl. 228 (1998), no. 1, 221-233. MR 2000j:76141. Zbl 0922.60054.

[3] G. Da Prato and J. Zabczyk, Stochastic Equations in Infinite Dimensions, Encyclopedia of Mathematics and Its Applications, vol. 44, Cambridge University Press, Cambridge, 1992. MR 95g:60073. Zbl 0761.60052.

[4]___Ergodicity for Infinite-Dimensional Systems, London Mathematical Society Lecture Note Series, vol. 229, Cambridge University Press, Cambridge, 1996. MR 97k:60165. Zbl 0849.60052.

[5] T. DelSole and B. F. Farrell, A stochastically excited linear system as a model for quasigeostrophic turbulence: analytic results for one- and two-layer fluids, J. Atmos. Sci. 52 (1995), 2531-2547. 
[6] J. Duan, P. E. Kloeden, and B. Schmalfuß, Exponential stability of the quasigeostrophic equation under random pertuabations, Proccedings Conference on Climate and Mathematics (Chorin 1999), Progr. Probab., vol. 49, Birkhäuser, Basel, 2001, pp. 241-256.

[7] V. Dymnikov and E. Kazantsev, On the "genetic memory" of chaotic attractor of the barotropic ocean model, Proceedings of the Third Bilateral Conference "Predictability of Atmospheric and Oceanic Circulation" of the French-Russian A. M. Liapunove Institute in Computer Science and Applied Mathematics (INRIA-Moscow State University), Nancy, 1997, pp. 25-36.

[8] L. C. Evans, Partial Differential Equations, Graduate Studies in Mathematics, vol. 19, American Mathematical Society, Rhode Island, 1998. MR 99e:35001. Zbl 0902.35002.

[9] B. Ferrario, Ergodic results for stochastic Navier-Stokes equation, Stochastics Stochastics Rep. 60 (1997), no. 3-4, 271-288. MR 98h:60090. Zbl 0882.60059.

[10] A. Griffa and S. Castellari, Nonlinear general circulation of an ocean model driven by wind with a stochastic component, J. Marine Research 49 (1991), 53-73.

[11] G. Holloway, Ocean circulation: flow in probability under statistical dynamical forcing, Stochastic Models in Geosystems (Minneapolis, MN, 1994), IMA Vol. Math. Appl., vol. 85, Springer, New York, 1997, pp. 137-148. CMP 1480973.

[12] P. Müller, Stochastic forcing of quasi-geostrophic eddies, Stochastic Modelling in Physical Oceanography (R. J. et al. Adler, ed.), Progr. Probab., vol. 39, Birkhäuser, Massachusettes, 1996, pp. 381-395. MR 97a:86007. Zbl 0865.76077.

[13] A. Pazy, Semigroups of Linear Operators and Applications to Partial Differential Equations, Applied Mathematical Sciences, vol. 44, Springer-Verlag, New York, 1983. MR 85g:47061. Zbl 0516.47023.

[14] J. Pedlosky, Geophysical Fluid Dynamics, 2nd ed., Springer-Verlag, New York, 1987. Zbl 0713.76005.

[15]__ Ocean Circulation Theory, Springer-Verlag, Berlin, 1996.

[16] R. M. Samelson, Stochastically forced current fluctuations in vertical shear and over topography, J. Geophys. Res. 94 (1989), 8207-8215.

[17] D. W. Stroock, Probability Theory: An Analytic View, Cambridge University Press, Cambridge, 1993. MR 95f:60003. Zbl 0925.60004.

Jingiao DuAn: Department of ApPlied Mathematics, Illinois Institute OF TeChNOLOGY, CHICAGO, IL 60616, USA

E-mail address: duan@i it.edu

Beniamin Goldys: School of Mathematics, The University of New South Wales, SYDNEY 2052, AUSTRALIA 


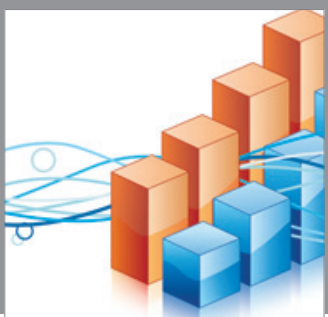

Advances in

Operations Research

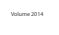

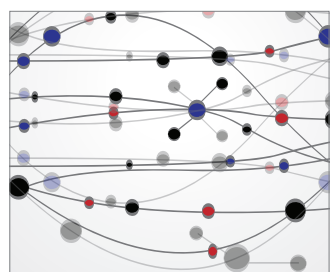

\section{The Scientific} World Journal
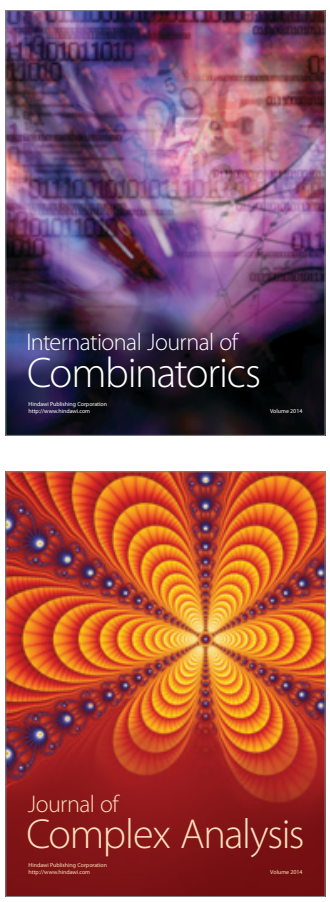

International Journal of

Mathematics and

Mathematical

Sciences
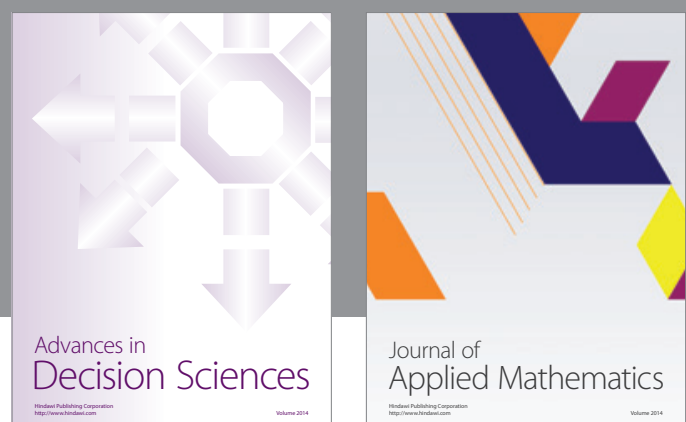

Journal of

Applied Mathematics
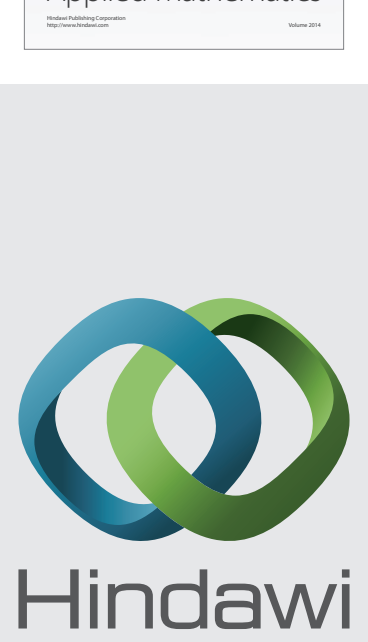

Submit your manuscripts at http://www.hindawi.com
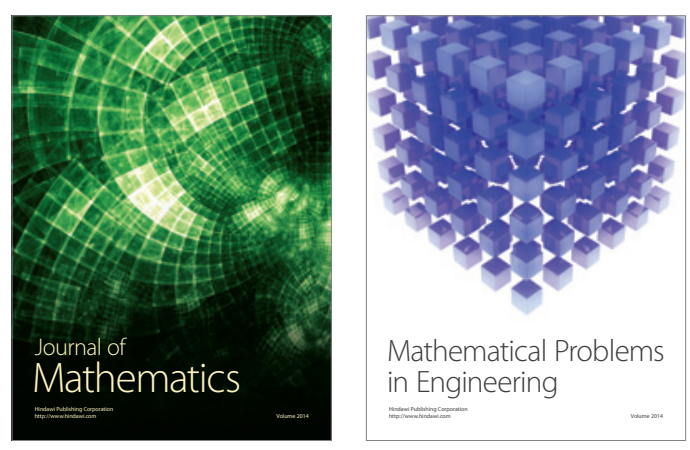

Mathematical Problems in Engineering
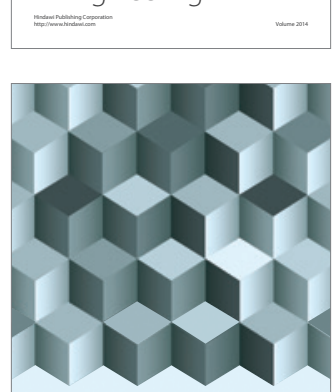

Journal of

Function Spaces
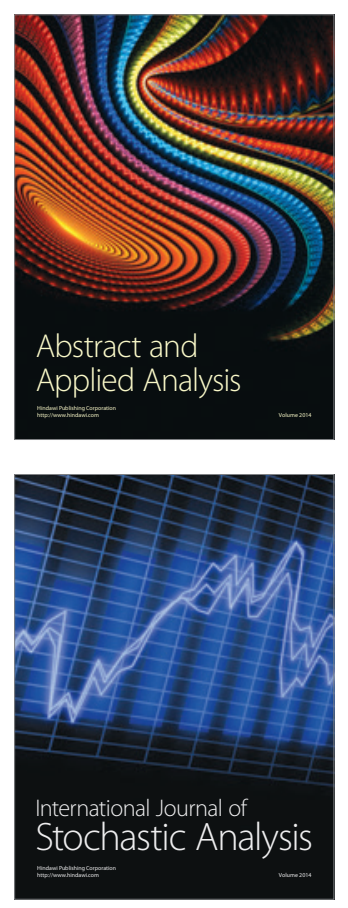

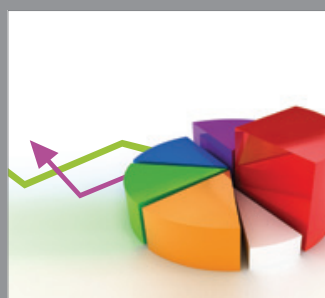

ournal of

Probability and Statistics

Promensencen
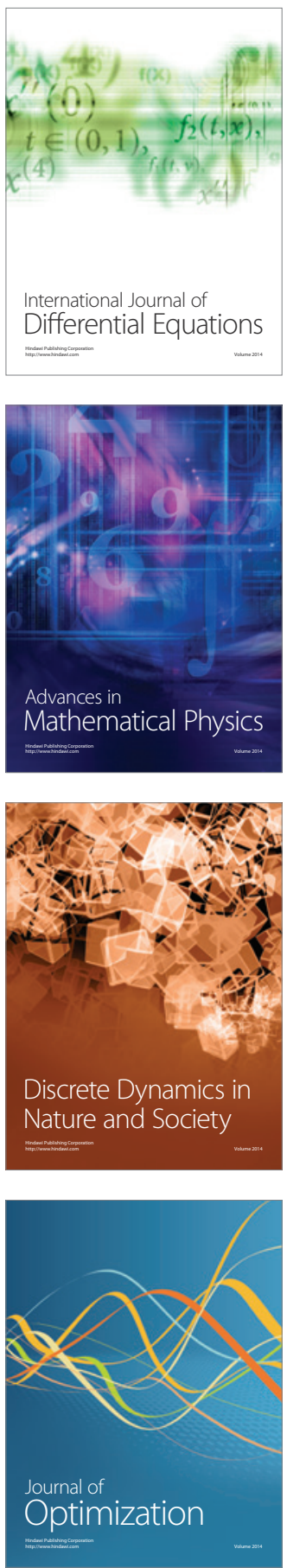